\title{
La reduplicación de las señas: un recurso gramatical importante en la investigación y en la enseñanza de la Lengua de Señas Cubana (LSC)
}

\author{
The reduplication of the sign: an important grammatical device in the research \\ and in the teaching of Cuban Sign Language (CSL)
}

\author{
Marianela Garau Cordovés ${ }^{1}$ \\ Universidad de La Habana (Cuba) \\ Eric Fernández Hernández ${ }^{2}$ \\ Universidad de La Habana (Cuba)
}

\begin{abstract}
- RESUMEN: Este artículo constituye una aproximación al estudio de un recurso gramatical de la LSC: la reduplicación de la seña. La propuesta surge a partir de los resultados de dos investigaciones cubanas. La primera de ellas es la caracterización del sustantivo como clase léxico-gramatical en la LSC (Garau, 2010), la cual enuncia en una de sus conclusiones que la reduplicación de la seña sustantiva constituye un importante recurso para la expresión del plural. La segunda investigación es un trabajo preliminar sobre las características que distinguen el verbo en la LSC (Suárez, Aragón, Garau, 2012). En su análisis, las autoras constataron que la reduplicación participa en la expresión de los aspectos continuativo e iterativo. El propósito principal es brindar una descripción que motive a los investigadores e instructores de la LSC a reflexionar sobre la importancia de este tema para las actuales descripciones lingüísticas y para el continuo perfeccionamiento de la enseñanza de la gramática de esta lengua.
\end{abstract}

- PALABRAS-CLAVES: Lengua de Señas Cubana (LSC); reduplicación de la seña; plural; aspecto; enseñanza de la LSC

- ABSTRACT: This article constitutes an approach to the study a grammatical resource of 'Cuban Sign Language (CSL): reduplication of the sign. The proposal rise up from the results of two Cuban researches. The first one is the characterization of nouns as type of word in CSL (Garau, 2010), which state in one of its conclusions that the reduplication of the substantive sign constitutes an important resource to express plural. The second research is a preliminary project about the characteristics that distinguish a verb in CSL (Suárez, Aragón, Garau, 2012). In its analysis, the authors state that the reduplication take part in the expression of continued and interactive aspects. The main purpose is to provide a description that motivates researchers and instructors of CSL to think about the importance of this issue for the current linguistic description and for the continuous improvement of the teaching of grammar in this language.

- KEYWORDS: Cuban Sign Language (CSL); reduplication of the sign; plural; aspect; teaching of CSL

\section{Introducción}

En 1960 ocurre un hecho considerado como el inicio de las descripciones lingüísticas de las lenguas de señas: Williams C. Stokoe, profesor de inglés de

\footnotetext{
${ }^{1}$ Autor principal. Maestra en Lingüística Aplicada por la Universidad de La Havana (Cuba). mgarau@,flex.uh.cu

${ }^{2}$ Orientador de la investigación. Prof. Titular y Consultante. Universidad de La Habana (Cuba). Dirección Relaciones Internacionales. Ministerio de Educación Superior de Cuba. ericflex@gmail.com
} 
estudiantes Sordos $^{3}$ en la Universidad de Gallaudet en Washington DC utilizó por primera vez, principios de la lingüística moderna para analizar la lengua utilizada por sus estudiantes: la Lengua de Señas Americana (ASL, por sus siglas en inglés). En este mismo año publicó su trabajo monográfico Sign Languag estructure: Anout line of the visual communications y stemof the American deaf, (Estructura de la lengua de signos: Primer análisis de la Lengua de Signos Americana) y cinco años más tarde, un primer diccionario, escrito junto con dos colegas Sordos.

Rocío Martínez (2016, p. 58) plantea que las mencionadas publicaciones permitieron demostrar que las señas podían ser analizadas internamente como mismo se hacía con las palabras en las lenguas orales, ya que Stokoe determina tres aspectos que se combinan de manera simultánea: el lugar de la articulación (tabula o tab, usualmente conocido como ubicación o locación), la forma de la mano (designator o dez, denominado normalmente configuración manual) y la acción de producir la seña (signation o sig, conocido como movimiento). La autora precisa, también, que la orientación de la palma de la mano y los rasgos no manuales, considerados hoy como otros aspectos, se describen en sistemas posteriores.

De acuerdo con Esperanza Morales (2000), en la década de los setenta surge la investigación sistemática sobre las lenguas de señas con el objetivo primordial de plantearse la realidad psicológica de diferentes aspectos de la organización estructural de estos sistemas. La autora precisa, que, de esta manera, prevalecía la tradición que, para las lenguas orales había comenzado en la década de los sesenta, con el intento de demostrar la validez empírica de los modelos lingüísticos propuestos hasta ese momento. La teoría gramatical de Noam Chomsky (1965), que postula la realidad cognitiva de una gramática universal, como resultado de la facultad del lenguaje innata en el ser humano, fue uno de los modelos imperantes en los análisis de esta primera etapa de investigación lingüística de las lenguas de señas, según plantea Morales (2000).

La mencionada autora apunta que, bajo la influencia de estos principios, los resultados de las investigaciones corroboraron para las lenguas de señas, las teorías postuladas hasta ese momento para las lenguas orales. Estas son:

1. la discutida existencia de una facultad innata para el lenguaje humano, independiente de la modalidad del canal utilizado; las diferencias solo se consideran por las características propias de cada canal: el oral y el visual.

2. la coincidencia en el desarrollo del lenguaje para ambos tipos de lenguas; los niños que aprenden una lengua oral y los que aprenden una lengua de señas transitan por las mismas etapas porque conciben ambas lenguas como un conjunto de unidades discretas en las que prevalecen las propiedades simbólicas sobre las icónicas.

3. la confirmación de que el hemisferio izquierdo es el detonante principal de la facultad del lenguaje para ambas modalidades.

\footnotetext{
${ }^{3}$ Por convención, la palabra Sordo se escribe con mayúsculas para referirse a la identidad lingüística y cultural de las personas que pertenecen a la comunidad Sorda (concepción socio-antropológica) y diferenciarse de la sordera como una condición audiológica (concepción clínica) (Cf. PADDEN, 1983 [1980]: 90; WILCOX, 2007: 1113; PFAU, STEINBACH Y WOLL, 2012) (Citados por MARTÍNEZ, 2016, p. 17). De igual manera, por convención, los nombres de todas las lenguas de señas se escriben con letras iniciales mayúsculas.
} 


\section{4. la confirmación de que en el proceso evolutivo humano se produce una readaptación del cerebro que permite desarrollar una lengua en un canal de trasmisión-recepción diferente.}

Con estos resultados, se constata científicamente que las lenguas de señas constituyen verdaderos sistemas lingüísticos y, además, comienza a establecerse una nueva disciplina: la lingüística de las lenguas de señas.

Como mencioné antes, Esperanza Morales (2000) define una segunda etapa (década de los noventa), en la que comienza un cambio de perspectiva teórica y metodológica en el análisis de las lenguas de señas por parte de algunos de sus investigadores. Considero que este hecho se debe a que se comienzan a enfrentar los estudios desde la propia realidad viso-gestual de las lenguas de señas y no desde la demostración, a ultranza, de que la mayoría de las teorías lingüísticas que fundamentan la estructura y el funcionamiento de las lenguas orales, pueden aplicarse directamente a las lenguas de señas.

En este sentido, Martínez (2016:61) destaca que, en esta etapa, determinados estudiosos comienzan a publicar trabajos sobre la base de los paradigmas de la lingüística sistémico-funcional, la lingüística cognitiva, los enfoques socioantropológicos, el enfoque comunicativo, etc. La referida autora cita las investigaciones sobre temas, tales como: iconicidad cognitiva (WILCOX, S. 1993, 2002A Y 2004; CUXAC, 1996 Y 2000; PIZZUTO Y VOLTERRA, 2000; PIETRANDREA, 2002), interacción entre gestos y señas (ARMSTRONG, STOKOE Y WILCOX, 1995; WILCOX, S. 2002B; LIDDELL, 2003; ARMSTRONG Y WILCOX, 2007), metonimia y metáfora conceptual (WILCOX, P., 2000; TAUB, 2001; JARQUE, 2005).

En el caso de Cuba, las investigaciones que tuvieron como objeto de estudio la descripción de la LSC, se iniciaron en el año 2003.Las primeras registradas: Valenciaga (2003, 2005) y Pérez (2004), se dirigieron al estudio de los parámetros formativos quinésicos de la lengua de señas cubana. En estos estudios se enunciaron, por primera vez en Cuba, la importancia de estos componentes mínimos desde un punto de vista lingüístico. El primer estudio realizado por Valenciaga advierte la necesidad de conocer y comprender el valor de los mencionados componentes para el proceso de enseñanza y aprendizaje de la LSC. Posteriormente, la investigadora profundizó en el conocimiento y la descripción de las configuraciones manuales (forma que adopta la mano para articular la seña) más frecuentes de la LSC.

Pérez (2004), por su parte, describe la seña a partir de los parámetros formativos quinésicos considerando que son aislables y tienen, en muchos casos, valor diferencial con múltiples variantes, al entrar en una combinación simultánea en el espacio. Los parámetros descritos son: configuración manual, lugar de articulación y movimiento. En el primero registra tres elementos importantes: región de contacto de la mano durante el movimiento, orientación de la configuración manual según la palma de la mano y la disposición, según el uso de las manos y las reglas básicas de simetría y asimetría de estas.

Padilla (2007) continúa la misma línea investigativa y define los cuatro componentes manuales (parámetros formativos quinésicos para las investigadoras anteriores) que conforman la seña (configuración manual, orientación de la(s) mano(s), locación de la(s) mano(s) y el movimiento). Presenta, además, una propuesta de bases teóricas y metodológicas para caracterizar la expresión del significado de estos componentes, estructurada por un sistema de dimensiones e indicadores que contiene: el espacio gestual, el uso de las manos, la situación comunicativa, la consideración distintiva de cada componente y la relación sistémica que se produce entre ellos para 
conformar las señas. Con una amplia descripción Padilla (2007), concreta la expresión del significado de: 24 configuraciones, 11 orientaciones de la mano, 6 locaciones de la mano en el espacio y 4 zonas en el cuerpo en las que se ubican las manos en las señas que se realizan cerca o en contacto con él, y 2 tipos de movimiento. Este último componente con una pormenorizada caracterización de 10 direcciones que asumen las manos, entre otros indicadores.

En el año 2005, con la apertura de la licenciatura en Interpretación de la lengua de señas en el departamento de Español de la Facultad de Lenguas Extranjeras (FLEX) perteneciente a la Universidad de La Habana y la incorporación de las dos profesoras principales a la Maestría en Lingüística aplicada desarrollada en dicho centro, se definieron temas de investigación para el estudio lingüístico de la LSC. Como resultado de este trabajo se obtuvieron las primeras descripciones de la expresión de la temporalidad (SALGADO, 2010) y de la seña sustantiva como clase léxico-gramatical (GARAU, 2010). Asimismo, las tesis de licenciatura del primer grupo de estudiantes profundizaron en el valor distintivo del componente movimiento (CALDERÓN, 2010) y el valor distintivo de la expresión facial (VIEL, 2010).

En las tesis de la carrera se investigaron: los elementos que conforman el nivel querológico (se refiere al nivel fonológico) (COTO, 2013), el sistema verbal -estudio preliminar- (Suárez, 2013) y las preposiciones (primera caracterización) (FERNÁNDEZ, 2013). Además, Calderón continuó el estudio del valor distintivo del componente movimiento en su relación con el espacio gestual y presentó su propuesta como tesis de maestría en la FLEX, también en el año $2013^{4}$.

En el presente artículo centro mi análisis en uno de los resultados de mi tesis de maestría "Caracterización del sustantivo como clase léxico-gramatical en la LSC" (GARAU, 2010), mencionada antes, y en un estudio preliminar sobre las características que distinguen el verbo en la LSC (SUÁREZ, ARAGÓN, GARAU, 2012).

\section{Estudio dela reduplicación en las lenguas de señas}

La sistematización de los estudios de las lenguas de señas permite afirmar que la reduplicación es un recurso gramatical que se observa en todas ellas. Padilla (2007, p. 80) hace referencia a ello por primera vez en Cuba al caracterizar el componente mínimo movimiento ${ }^{5}$. Específicamente en el movimiento externo, define la modalidad como una de sus dimensiones:

\footnotetext{
${ }^{4}$ En la actualidad, en el Centro de Superación y Desarrollo del Sordo perteneciente a la Asociación Nacional de Sordos de Cuba (ANSOC) radica el Grupo de Investigación de la LSC (GILESC), está constituido por un colectivo de profesores, quienes continúan las investigaciones de la LSC. Su colaboración ha sido esencial para los resultados que se enunciaron anteriormente. Este centro es también, rector de los estudios de registro léxico de la LSC desde la década de los noventa. Asimismo, en él se realizan investigaciones dirigidas al campo de la interpretación, las particularidades socioculturales de la comunidad Sorda cubana y la didáctica de la enseñanza de la LSC.

${ }^{5} \mathrm{El}$ término componentes responde a la terminología cubana; pero las Gramáticas de otras lenguas de señas (Lengua de Señas Colombiana, Lengua de Señas Argentina, Lengua de Señas Uruguaya, Lengua de Signos Española, Lengua de Señas Mexicana, Lengua de Signos Catalana, entre otras, designan el movimiento, la orientación, la locación y la configuración manual como unidades fonológicas. Esto se debe a la tendencia actual de los investigadores de la disciplina a considerar el carácter abstracto y no el material del fonema. En este sentido, aunque esta idea está ampliamente difundida, los integrantes del GILESC mantienen la terminología de unidades queirológicas o quieremas de la década de los sesenta. Debido a que las investigaciones descriptivas de la LSC, a mi juicio, todavía carecen de precisiones teóricas, he preferido llamarlos "componentes mínimos".
} 
La modalidad ${ }^{6}$ del movimiento, Indica cómo se describe o transcurre la acción. Se caracteriza la expresión del significado atendiendo a cuatro indicadores fundamentales:

- Velocidad: significa la rapidez, agilidad, aceleración o lentitud y pasividad con que se realiza la acción. En muchos casos transparentan la realidad, es decir, se describe tal y cómo ocurre la acción.

- Frecuencia: denota cómo se reproduce la acción, con periodicidad, continuada, reiterada, diversa o irregular. Significa la reduplicación en mayor o menor medida de la acción.

- Intensidad: significa fuerza y energía, también violencia, vigor, énfasis, firmeza, potencia y poder.

- Magnitud: significa la dimensión o extensión del movimiento, el volumen, capacidad, grosor, influencia, cuantía, proporción y tamaño.

Por ejemplo: las señas ola, beso, flor, pueden variar su significado al realizar varias veces del mismo movimiento, es decir, con frecuencia. Este caso se indica una pluralidad y la expresión sería olas, besos, flores (PADILLA, 2007, p. 80).

En la cita anterior se constata que la autora alude a la reduplicación de la seña para la expresión del plural. Aunque no lo enuncia como un recurso morfológico, considero que esta definición constituye un antecedente significativo del tema.

Posteriormente, Garau (2010, p. 46) incluye la reduplicación en el análisis morfológico de la seña sustantiva y constata que constituye un recurso que permite expresar el plural. Los siguientes ejemplos ilustran las formas singulares CASA, FLOR, SILLA (fig.1). Con la reduplicación de ellas en el espacio gestual ${ }^{7}$ se expresan las formas en plural CASAS, FLORES, SILLAS.

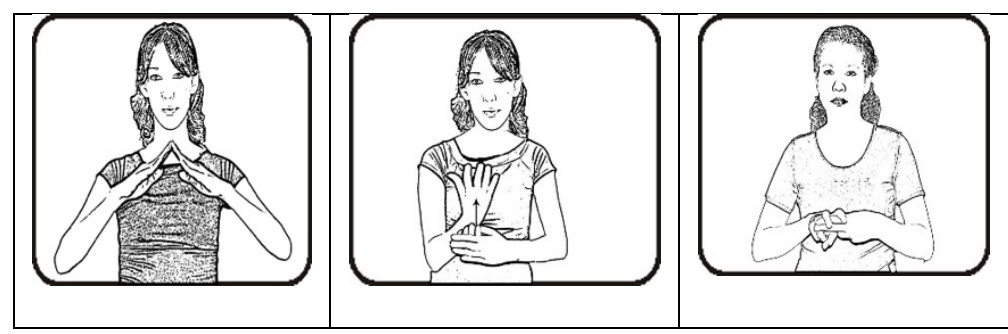

Figura 1. Las señas CASA, FLOR y SILLA de la LSC en su forma singular.

La reduplicación de señas como las anteriores reproduce, en el espacio gestual, una analogía de la disposición que generalmente poseen los objetos en el espacio físico. A este mecanismo se le ha denominado plural descriptivo. (HERRERO, 2009, p. 186). Asimismo, sucede con las señas sustantivas que se articulan haciendo contacto con el cuerpo. La figura 2 muestra la forma singular de BESOS, AÑOS, CADENA (prenda). La reduplicación puede realizarse en la misma locación corporal o en diferentes locaciones para significar, por ejemplo: besos por todo el cuerpo o botones en las

\footnotetext{
${ }^{6}$ Cabe destacar que la modalidad del movimiento no es un término apropiado para esta definición, en tanto alude a rasgos referenciales, tales como: velocidad, frecuencia, intensidad y magnitud. Considero que es necesario revisar este aspecto en investigaciones posteriores y tener en cuenta, además, que modalidad es un término "ocupado" dentro de la lingüística.

${ }^{7}$ El espacio gestual, es el área requerida para la conformación de las señas y tiene un carácter tridimensional. La amplitud del espacio está relacionada directamente con el emisor y comprende un espacio horizontal y uno vertical. La amplitud del espacio horizontal es de 180 grados; abarca toda el área recorrida por el desplazamiento de los brazos del emisor, semi-flexionados a la altura de la cintura, desde el frente hacia ambos lados del cuerpo. La amplitud del espacio vertical es de 90 grados; abarca el área recorrida por el desplazamiento de los brazos del emisor, semiflexionados al nivel de la cintura, desde el frente hasta la altura de la cabeza (PADILLA, 2007, p. 51).
} 
mangas, en el cuello, en los bolsillos. Considero que estos constituyen, también, casos de plural descriptivo.

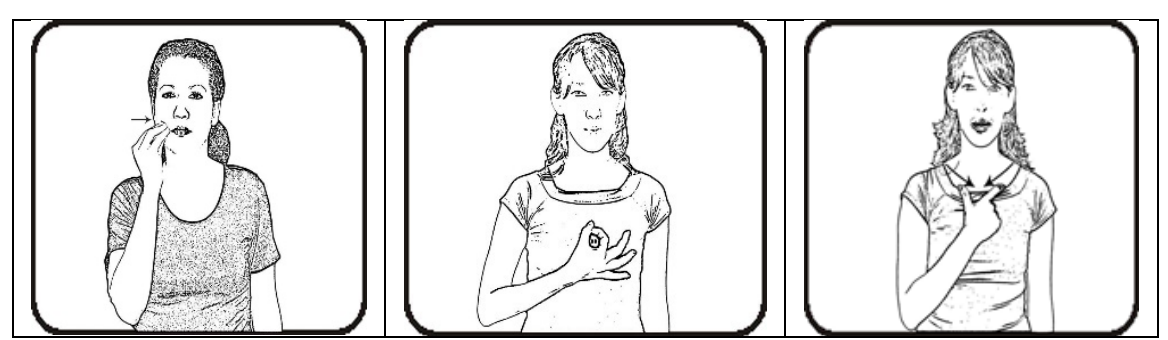

Figura 2. Las señas BESO, AÑO, CADENA (prenda) de la LSC en su forma singular

Las dos autoras cubanas coinciden en que la reduplicación es un recurso que permite expresar el plural en la LSC. La primera llega a esta conclusión a partir de un exhaustivo análisis que define cuáles son los componentes mínimos en la LSC; y la segunda, al tener como objetivo caracterizar la seña sustantiva como clase-léxicogramatical, comprueba que este recurso modifica la seña, por tanto: es un proceso morfológico que permite expresar el plural.

Cabe señalar que, en la actualidad, las investigaciones de las lenguas de señas incluyen las expresiones de pluralidad en un concepto más abarcador: la cuantificación, entendida como los recursos que poseen las lenguas para expresar la cantidad en sentido amplio.

Otras investigaciones han demostrado que la reduplicación de las señas constituye también un procedimiento productivo para la expresión de contenidos aspectuales. Diyer (1976), Fisher y Bonnie (1978), Klima y Bellugi (1979), Kiley y Woll (1985), Rodríguez (1992), Amaral, Couthino y Delgado (1994) (Citados por FREIRE, 2000, p. 76).

Cristina Freire Rodríguez, en su tesis "Bilingüismo lengua de signos - lengua oral: consideraciones en torno al aspecto en lengua de signos española (LSE) y en español escrito", realiza un análisis exhaustivo de cómo ha sido estudiada esta categoría tanto en las lenguas orales como en las de señas. Su objetivo es establecer una propuesta de tipología aspectual para la LSE.

La autora describe los procesos morfológicos, los sintácticos; y en algunos casos, los modificadores oracionales y mecanismos pragmáticos que permiten la expresión del aspecto en la variante de LSE correspondiente a la zona de La Coruña. Dentro de los primeros aparece la reduplicación de la seña en la expresión de los aspectos continuativo, durativo, iterativo y distributivo.

En una aproximación a las características que distinguen el verbo en la lengua de señas cubana Suárez, Aragón, Garau (2012) coinciden con el análisis de Freire (2000) y toman como referencia sus definiciones para ejemplificar, por primera vez en Cuba, la expresión de los aspectos continuativo e iterativo en la LSC. Para este trabajo se han escogido, solamente los ejemplos que contienen la reduplicación.

Según Freire, "el aspecto continuativo focaliza la acción en su etapa intermedia y a menudo se solapa con el durativo, el cual se centra en el tiempo empleado en la ejecución de una acción". Ella considera la reduplicación como recurso morfológico más frecuente. Los siguientes ejemplos muestran las transcripciones de cuatro construcciones simples de la LSC con verbos que incluyen esta información aspectual, pues la reduplicación indica que las acciones persisten en un intervalo de tiempo. 
PROFESOR EXPLICAR (con reduplicación de la seña verbal)

REBECA COMER (con reduplicación de la seña verbal)

RICHARD JUGAR (con reduplicación de la seña verbal)

ARIEL TRABAJAR (con reduplicación de la seña verbal)

Las autoras cubanas constataron que la articulación de algunos verbos que admiten aspecto continuativo-durativo, se acompaña de una expresión facial (la lengua se coloca, ligeramente, fuera de la boca) que ofrece información de continuidad. Las fotos muestran las articulaciones de TRABAJAR y JUGAR con la inclusión de dicha expresión.

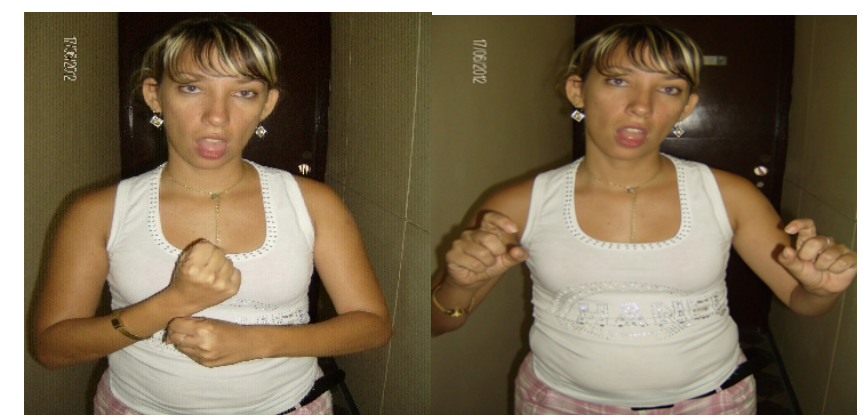

Figura 3. Señas TRABAJAR y JUGAR de la LSC

Freire (2000, p. 86) también define que "el aspecto iterativo remite a acciones que se repiten varias veces en un lapso de tiempo y que la reduplicación de la seña con pausas intercaladas, es un procedimiento morfológico indicador de esta información aspectual. Esto confiere la idea de que la acción se realiza en su totalidad dos o más veces". Los verbos de la LSC: COMPRAR, PAGAR, ARREGLAR, TRABAJAR, BUSCAR, HABLAR, EXPLICAR, ESCRIBIR, LEER, ESTUDIAR, ENSEÑAR, PEDIR, ENCONTRARSE, COPIAR, TOCAR una puerta, GASTAR dinero, incorporan esta información. El contenido que se trasmite se puede traducir como: compró muchas veces, habló en reiteradas ocasiones, pagó muchas veces, etc. En la siguiente foto se muestra el verbo PAGAR con la expresión de los labios que trasmite la repetición de la acción. La articulación del verbo se reduplica.

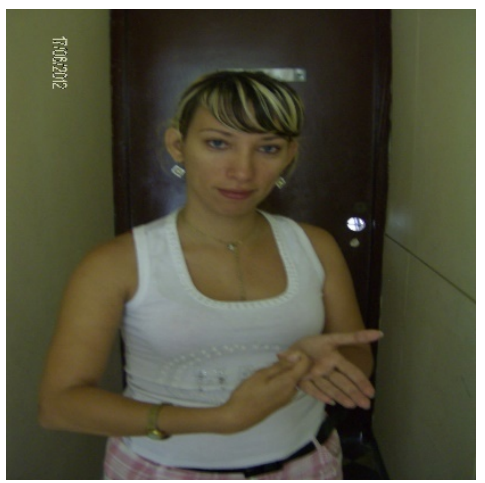

Figura 4. Seña PAGAR de la LSC

En los verbos de la LSC que incluyen información pronominal, pueden reduplicarse para indicar que la acción recae sobre el mismo beneficiario. La articulación de los ejemplos que aparecen a continuación va acompañada del 
movimiento realizado con la boca que refuerza la información aspectual que aparece en la imagen anterior.

AYUDAR (reduplicación hacia el lugar que ocupa la segunda persona ${ }^{8}$ en el espacio gestual) "Te ayudé en reiteradas ocasiones"

REGALAR (reduplicación hacia el lugar que ocupa la tercera persona en el espacio gestual) "Le regalé una y otra vez"

RESPONDER (reduplicación hacia el lugar que ocupa la segunda persona en el espacio gestual) "Te respondo muchas veces"

DAR (reduplicación hacia el lugar que ocupa la tercera persona en el espacio gestual)

"Te lo doy en reiteradas ocasiones"

ACONSEJAR (reduplicación desde el lugar que ocupa la tercera persona en el espacio gestual hacia el emisor) "Ella me aconsejó una y otra vez"

DECIR (reduplicación desde el lugar que ocupa la tercera persona en el espacio gestual hacia el emisor): "Él me lo dijo muchas veces"

Como el recurso morfológico es la reduplicación de la seña verbal, es necesario esclarecer que, en dependencia de la predicación, los verbos, contienen una u otra información aspectual. Por ejemplo:

ESCRIBIR (reduplicación de la seña verbal que muestra la continuidad de la acción con apoyo de expresión facial) "Estoy escribiendo"

ESCRIBIR (reduplicación de la seña verbal que muestra la reduplicación de la acción en un intervalo de tiempo con apoyo de expresión facial y un gesto con las manos que indica mucho) "Escribí muchas veces"

\section{Consideraciones finales}

Si se analizan las señas con reduplicación, presentadas en los ejemplos de este artículo, se puede constatar que estas poseen determinadas características:

- Se articulan con una sola mano: BESO, AÑO, CADENA (prenda), PALABRA, EXPLICAR, DAR, PEDIR, HABLAR, COMPRAR.

- las bimanuales simétricas, es decir, las que poseen todos sus componentes mínimos iguales en ambas manos: CASA, TRABAJAR y JUGAR.

- algunas señas bimanuales no simétricas, es decir, que se articulan con las dos manos; pero una, se mantiene activa y la otra, pasiva: AYUDAR, ESCRIBIR, PAGAR.

Tener en cuenta que la reduplicación es un recurso morfológico que permite la expresión de categorías gramaticales tales como la pluralidad y el aspecto, abre un camino para la investigación sobre la productividad de dicho recurso en la LSC.

${ }^{8}$ En el espacio gestual o sígnico se destina una locación para cada persona gramatical. 
Además, la consideración de que ciertas características de las señas condicionan su aparición, indica la existencia de otros recursos para expresar las mismas categorías en otro tipo de señas. Por ejemplo, MAMÁ y PAPÁ, que son monomanuales y en su realización ya poseen un movimiento repetido, no admiten la reduplicación. Es necesario investigar los recursos que permiten expresar la idea de plural en "muchas madres" o "muchos padres".

En relación con la enseñanza, es de vital importancia someter estos resultados preliminares al análisis de los instructores Sordos, pues considero que el intercambio con ellos potencia la introducción de aspectos gramaticales, como los tratados en este trabajo, en los programas actuales de enseñanza de la LSC.

\section{BIBLIOGRAFÍA}

BARÓN BIRCHENALL, L. y MÜLLER, O. La Teoría Lingüística de Noam Chomsky: del Inicio a la Actualidad. Lenguaje, Colômbia, v. 42, n. 2, p. 417-442, 2014.

CALDERÓN VERDE, A. El valor distintivo del componente manual movimiento de la Lengua de Señas Cubana en su relación con el espacio gestual (Tesis de licenciatura). La Habana. Facultad de Lenguas Extranjeras. Universidad de La Habana. Cuba, 2010.

El valor distintivo del componente manual movimiento de la Lengua de

Señas Cubana (Tesis de maestría). La Habana. Facultad de Lenguas Extranjeras. Universidad de La Habana. Cuba, 2013.

Colectivo de autores de la Asociación de Sordos de Cuba. Manual de Lengua de Señas Cubana. Tomo II. HANDICAP. INTERNACIONAL, 2010.

COTO CARTAYA, E. Los componentes manuales del nivel querológico en la Lengua de Señas Cubana: elementos que lo conforman (Trabajo de diploma). La Habana. Facultad de Lenguas Extranjeras. Universidad de La Habana. Cuba, 2013.

CRUZ ALDRETE, M. Gramática de la Lengua de Señas Mexicana (Tesis de doctorado). D. F., Centro de Estudios Lingüísticos y Literarios. México, 2008.

CURBEIRA CANCELA, A. Lecturas de Semántica I. La Habana: Editorial Félix Varela, 2003. 2007.

Introducción a la Teoría del lenguaje. La Habana: Editorial Félix Varela,

FERNÁNDEZ SONEIRA, Ana Ma. (2008). La cantidad a manos llenas. La expresión de la cuantificación en la lengua de signos española, 2008. Disponible en http//www.culturasorda.

FREIRE RODRÍGUEZ, C. Bilingüismo lengua de signos - lengua oral: consideraciones en torno al aspecto en lengua de signos española (LSE) y en español escrito. Tesis. Coruña. Universidad A Coruña. España, 2000.

GARAU CORDOVÉS, M. Caracterización del sustantivo como clase léxico gramatical en la Lengua de Señas Cubana (Tesis de maestría). La Habana. Facultad de Lenguas Extranjeras. Universidad de La Habana, Cuba, 2010.

HERRERO BLANCO, A. Gramática didáctica de la lengua de signos española. Madrid: Ediciones SM, 2009.

MARTÍNEZ, R. A. Reconsideración, desde un Enfoque Cognitivo-Prototípico, del adjetivo como clase de palabras en la Lengua de Señas Argentina (LSA) (Tesis de doctorado). Buenos Aires. Facultad de Filosofía y Letras. Argentina, 2016.

MASSONE, M. I. y MACHADO, E. Lengua de Señas Argentina. Análisis vocabulario bilingüe. Buenos Aires: EDICIAL S.A, 1994. 
MASSONE, M. I.; CURIEL, M.; BUSCAGLIA, V.; FAMULARO, R.; SIMÓN, M.; CARBONI, I. La Conversación en la Lengua de Señas Argentina. Buenos Aires: Edicial-Libros en Red, 2000.

MASSONE, M. I.; MARTÍNEZ, R.A. Curso de Lengua de Señas Argentina, 2012. Disponible en http//www.cultura-sorda.eu

MORALES LÓPEZ, E. Lingüística de las Lenguas de Signos: perspectiva histórica. Coruña: Universidad de Coruña, 2000. Disponible en http//ww.cervantesvirtual.com OVIEDO, A. Un estudio sobre la estructura de las señas de la Lengua de Señas Venezolana. Mérida, Venezuela: Universidad de Los Andes, 2000.

Apuntes para una gramática de la Lengua de Señas Colombiana. Santiago de Cali, Colombia: Universidad del Valle, 2001.

PADILLA FERNÁNDEZ, L. (2007). El significado de los componentes manuales de la Lengua de Señas Cubana como sistema lingüístico (Tesis de maestría). La Habana. Facultad de Educación Infantil. Universidad Pedagógica Enrique José Varona. Cuba, 2007.

PÉREZ NIEVES, H. Estudio lingüístico de los parámetros formativos quinésicos de la lengua de señas de los escolares sordos, en el área de Química. (Trabajo de diploma). La Habana. Universidad Pedagógica Enrique José Varona. Cuba, 2004.

RAYA HERNÁNDEZ, M. G.; ZULUETA BLANCO, M. E. Textos científicotécnicos. ¿Cómo crearlos? La Habana: Editorial científico-técnica, 2001.

SUÁREZ MARRERO, M., ARAGÓN FEBLES; GARAU CORDOVÉS, M. Características que distinguen al verbo en la lengua de señas cubana (Trabajo de curso). La Habana. Facultad de Lenguas Extranjeras, Universidad de La Habana, 2012.

VIEL FERNÁNDEZ, T. El valor distintivo de la expresión facial como componente no manual en la Lengua de Señas Cubana (Trabajo de diploma). La Habana . Universidad de La Habana, Cuba, 2010.

Recebido em: julho de 2018.

Aprovado em: agosto de 2018.

Como citar este trabalho:

CORDOVÉS, M. G.; HERNÁNDEZ, E. F. La reduplicación de las señas: un recurso gramatical importante en la investigación y en la enseñanza de la Lengua de Señas Cubana (LSC). Traços de linguagem, v. 2, n.2, p. 61-70, 2018. 